\title{
Interaction between the ADAMTS-12 metalloprotease and fibulin-2 induces tumor-suppressive effects in breast cancer cells
}

\author{
Tania Fontanil1,2,*, Susana Rúa ${ }^{1,2, *}$, María Llamazares ${ }^{3}$, Angela Moncada-Pazos ${ }^{4}$, \\ Pedro M. Quirós ${ }^{1,2}$, Olivia García-Suárez 2,5, Jose A. Vega ${ }^{2,5}$, Takako Sasakí, Yamina \\ Mohamedi ${ }^{1,2}$, Manuel M. Esteban', Alvaro J. Obaya ${ }^{2,7}$ and Santiago Cal ${ }^{1,2}$ \\ ${ }^{1}$ Departamento de Bioquímica y Biología Molecular, Facultad de Medicina, Universidad de Oviedo, Asturias, Spain \\ 2 IUOPA, Instituto Universitario de Oncología del Principado de Asturias, Spain \\ ${ }^{3}$ German Cancer Research Center (DKFZ) Division Signal Transduction and Growth, Heidelberg, Germany \\ ${ }^{4}$ William Dunn School of Pathology, University of Oxford, Oxford, United Kingdom \\ ${ }^{5}$ Morfología y Biología Celular (grupo SINPOS), Facultad de Medicina, Universidad de Oviedo, Asturias, Spain \\ ${ }^{6}$ Department of Biochemistry, Faculty of Medicine, Oita University, Japan. \\ ${ }^{7}$ Biología Funcional, Facultad de Medicina, Universidad de Oviedo, Asturias, Spain \\ * These authors contributed equally to this work. \\ Correspondence to: Santiago Cal, email: santical@uniovi.es \\ Keywords: extracellular matrix, metalloprotease, ADAMTS, fibulin, breast cancer \\ Received: December 12, 2013 Accepted: January 12, $2014 \quad$ Published: January 12, 2014
}

This is an open-access article distributed under the terms of the Creative Commons Attribution License, which permits unrestricted use, distribution, and reproduction in any medium, provided the original author and source are credited.

\section{ABSTRACT:}

Balance between pro-tumor and anti-tumor effects may be affected by molecular interactions within tumor microenvironment. On this basis we searched for molecular partners of ADAMTS-12, a secreted metalloprotease that shows both oncogenic and tumor-suppressive effects. Using its spacer region as a bait in a yeast two-hybrid screen, we identified fibulin-2 as a potential ADAMTS-12-interacting protein. Fibulins are components of basement membranes and elastic matrix fibers in connective tissue. Besides this structural function, fibulins also play crucial roles in different biological events, including tumorigenesis. To examine the functional consequences of the ADAMTS-12/fibulin-2 interaction, we performed different in vitro assays using two breast cancer cell lines: the poorly invasive MCF-7 and the highly invasive MDAMB-231. Overall our data indicate that this interaction promotes anti-tumor effects in breast cancer cells. To assess the in vivo relevance of this interaction, we induced tumors in nude mice using MCF-7 cells expressing both ADAMTS-12 and fibulin-2 that showed a remarkable growth deficiency. Additionally, we also found that ADAMTS-12 may elicit pro-tumor effects in the absence of fibulin-2. Immunohistochemical staining of breast cancer samples allowed the detection of both ADAMTS-12 and fibulin-2 in the connective tissue surrounding tumor area in less aggressive carcinomas. However, both proteins are hardly detected in more aggressive tumors. These data and survival analysis plots of breast cancer patients suggest that concomitant detection of ADAMTS-12 and fibulin-2 could be a good prognosis marker in breast cancer diagnosis. 


\section{INTRODUCTION}

Extracellular matrix (ECM) components and their interactions are essential for homeostasis of tissues and provide structural support to animal cells [1-2]. These components also play crucial roles on cell dynamic behavior and act as modulators of intercellular communication [3-4]. Among them, fibulins family consists of seven secreted glycoproteins that share a globular domain at the carboxy-terminus, known as fibulin module, which is preceded by tandem calcium-binding Epithelial Growth Factor (cbEGF) domains [5-7]. Aminoterminal region displays the highest structural variability. Functionally fibulins serve as a scaffold for different ECM components including proteoglycans [8], fibronectin [9], collagen [10], fibrillin [11] or laminin [12]. In addition to this structural role, fibulins can also participate in complex biological processes including cell migration, adhesion and proliferation [13], and mediate cell signaling events [14-15].

Fibulin-2 illustrates an example for the contribution of fibulins to those processes. In fact, fibulin-2 is recognized as a multifunctional binding protein due to its ability to bridge diverse ECM components $[8,16]$. For instance, fibulin-2 participates in the assembling of the laminin network in the basement membrane through the binding of laminin- 1 and laminin-5 to other matrix proteins [17]. Also, binding of nidogen to fibulin-2 contributes to the formation of ternary complexes with type IV collagen, perlecan and fibulin-1 [18]. Moreover, fibulin-2 promotes cell-ECM contacts by binding to $\beta 3$ integrins [19]. These and other examples $[16,20]$ highlight the relevance of fibulin-2 in the maintenance of extracellular structures such as basement membranes, as well as contacts between cells and ECM. Complexity of functions of fibulin-2 is also evident in pathological conditions such as cancer [13, 21]. In this regard, pro-tumor effects have been associated to this fibulin in pancreatic cancer cells as consequence of its interaction with the transmembrane type I glycoprotein MUC4 [22]. However, a growing number of studies point out the anti-tumor properties of fibulin-2. For instance, this fibulin acts as an anti-angiogenic factor in nasopharyngeal carcinoma [23]; FBLN2, the human gene coding for fibulin-2, is found epigenetically silenced in B cell acute lymphoblastic leukemia [24]; and loss of fibulin-2 expression facilitates tumor progression in breast cancer cells [25]. These findings reveal the importance of fibulin-2 in tumor development. Nonetheless, the molecular mechanism by which this fibulin promotes or inhibits tumorigenesis is unclear at present.

ADAMTS-12 is a secreted metalloprotease [26] and different studies have suggested a role for this metalloprotease in tissue remodeling and cell migration or adhesion [27-28]. Moreover, ADAMTS-12 acts as a tumor-suppressor enzyme. Thus, this enzyme hinders anti-tumor effects in Madin-Darby canine kidney (MDCK) cells treated with hepatocyte growth factor [29]. Furthermore, ADAMTS12 gene promoter is hypermethylated in colorectal carcinomas [30]. Phenotypic analysis of the Adamts 12-deficient mouse has confirmed the tumor-protective role of this enzyme [31]. By contrast, ADAMTS-12 has been also associated to trophoblastic cell invasion by modulating the expression and function of $\alpha_{\mathrm{v}} \beta_{3}$ integrin [32]. Exploring the mechanisms that link ADAMTS-12 to tumorigenesis would help to minimize pro-tumor properties and thereby enhance anti-tumor effects of this metalloprotease.

Here we show that ADAMTS- 12 and fibulin-2 are two interacting proteins. Functional consequences of this interaction were evaluated using different cell-based assays. Two breast cancer cell lines, MCF-7 and MDAMB-231, were employed attending to the anti-tumor role of fibulin-2 [25] and expression profile of ADAMTS12 [33] in this type of tumor. Immunohistochemical detection of fibulin-2 and ADAMTS-12 was also performed using a human breast cancer tissue array. Main conclusion is that ADAMTS-12/fibulin-2 interaction potentiates anti-tumor effects in breast cancer cells.

\section{RESULTS}

\section{ADAMTS-12 and fibulin-2 are two interacting proteins}

Different fragments corresponding to the ADAMTS-12 exosites, including thrombospondins and spacer regions, were employed as baits to screen for molecular partners of the metalloprotease using yeast twohybrid assays. Among them, a fragment corresponding to spacer-1 allowed to select different clones containing inserts that according to BLAST analysis at NCBI (www. ncbi.nlm.nih.gov) matched with extracellular matrix proteins such as NELL2, connective tissue growth factor or agrin. Interestingly, one of these clones, with a 630bp insert, corresponded to nucleotides 3360 to 3990 of human fibulin-2 cDNA (GenBank ${ }^{\mathrm{TM}}$ accession number X82494.1). Conceptual translation of this region showed that the sequence matched with the carboxyl-end region of fibulin-2, including the final 88 amino acids of the fibulin module (Fig.1A). Taking in account that an interaction between fibulin-1 and ADAMTS-1 had been previously described [34], we decided to explore the functional consequences of a new interaction between an ADAMTS metalloprotease and a fibulin. As a first approach to validate this interaction we examined a potential co-immunoprecipitation of both proteins. To this end, we employed 293-EBNA cell extracts containing ADAMTS-12 tagged with a FLAG epitope. Different 
amounts of fibulin-2 were added to these extracts and immunoprecipitation was performed using an anti-FLAG M2 affinity gel. Immunoprecipitates were visualized by Western blot using an anti-fibulin-2 antibody (Fig. 1B). An immunoreactive band corresponding to fibulin-2 was detected in those cell extracts containing ADAMTS-12 incubated with the fibulin. However this band was not detected without incubation with fibulin-2 or when the same immunoprecipitation procedure was applied to extracts of EBNA control cells (EBNAc) (Fig. 1B).

A

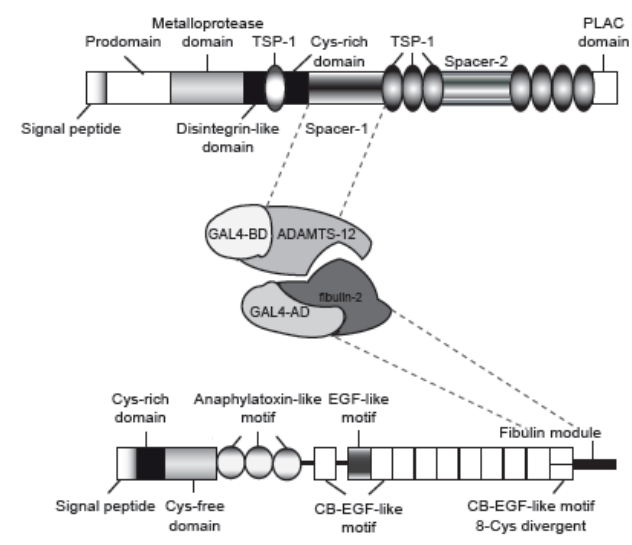

B

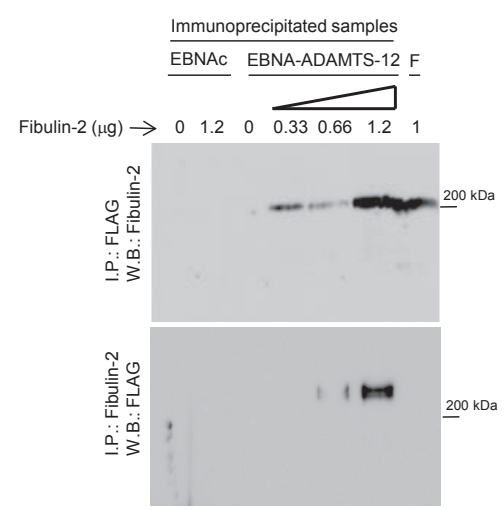

Figure 1: ADAMTS-12 and fibulin-2 are two interacting proteins. (A) Schematic illustration of the interacting regions between ADAMTS-12 and fibulin-2 determined by yeast twohybrid screen. TSP-1, thrombospondin-1-like domain; Cysrich domain, cysteine-rich domain; PLAC, protease-lacunin; RGD, Arginine-Glycine-Aspartic Acid; EGF, ephitelial growth factor; CB-EGF, Calcium-binding EGF; BD, binding domain; $\mathrm{AD}$, activating domain. (B) Western blot analysis of immunoprecipitated samples. Top, 293-EBNA control cells extracts (EBNAc) or 293-EBNA cell extracts producing ADAMTS-12-FLAG (EBNA-ADAMTS-12) were incubated with the indicated amounts of fibulin-2, immunoprecipitated with using anti-FLAG M2 affinity gel and further detection was performed with an anti-fibulin-2 antibody. Bottom, cell extracts were immunoprecipitated with an anti-fibulin-2 antibody and detection was performed with an anti-ADAMTS-12 antibody. $\mathrm{F}$ indicates that $1 \mu \mathrm{g}$ of fibulin-2 was loaded in these lanes. Molecular weight marker is indicated on the right.
Conversely, cell extracts were also immunoprecipitated with an anti-fibulin-2 antibody and immunoblotted with an anti-FLAG antibody. In this case, an immunoreactive band corresponding to ADAMTS-12 was detected in two samples containing fibulin-2, but not in control samples (Fig. 1B). These results strongly suggest that fibulin-2 physically binds to ADAMTS-12. Considering this, we decided to examine whether fibulin- 2 could act as a cofactor for the metalloprotease, similarly to what occurs with fibulin-1 and ADAMTS-1 [34]. However, presence
A

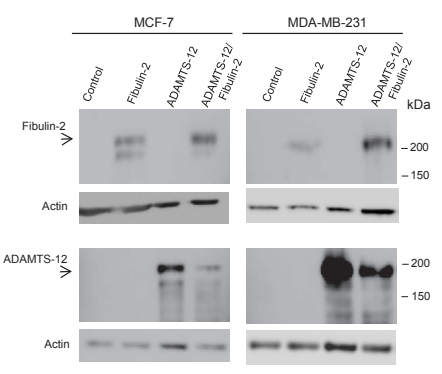

C

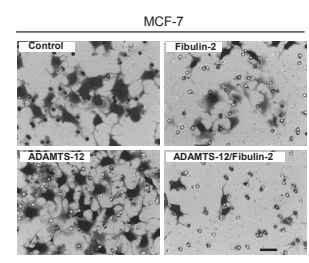

MDA-MB-231

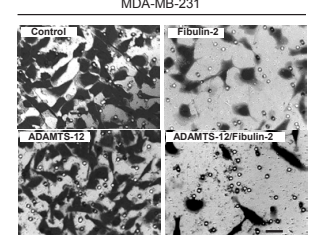

B

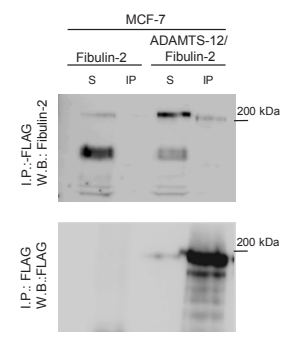

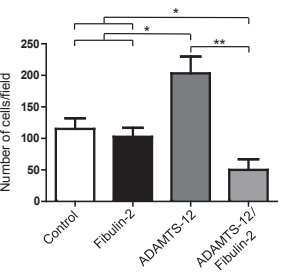

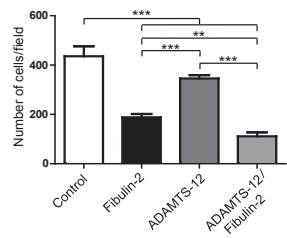

Figure 2: Selection of MCF-7 and MDA-MB-231 stable transfectants and invasion assay. (A) Western blot analysis of MCF-7 and MDA-MB-231 producing exogenous fibulin-2, ADAMTS-12 or both proteins simultaneously (ADAMTS-12/ fibulin-2). These cells are referred as (fb), (ts) and (fb/ts) respectively in the body text. Control, cells transfected with an empty vector. Top, detection with an anti-fibulin-2 antibody. Bottom, detection with an anti-FLAG (ADAMTS-12) antibody. Samples were run in separated gels due to the similar molecular weights of fibulin-2 and ADAMTS-12. Actin was used as a loading control. Molecular weight markers are indicated on the right. (B) Immunoprecipitation of MCF-7 cell extracts producing fibulin-2 or both fibulin-2 and ADAMTS-12 using anti-FLAG M2 affinity gel. S, cell extracts. IP, immunoprepitated. Top, detection with an anti-fibulin-2 antibody. Bottom, detection with an anti-FLAG (ADAMTS-12) antibody. (C) Cell invasion assay using Matrigel-coated invasion chambers. Representative microscopic pictures of MCF-7 (top) and MDA-MB-231 (bottom) cells producing exogenous fibulin-2, ADAMTS-12 or both proteins simultaneously. Cells transfected with an empty vector were used as control. Cells that reached the lower surface were counted and graphically represented. Scale bar: $50 \mu \mathrm{m}$. 
of fibulin-2 did not increase the low aggrecanase activity of ADAMTS-12 using a peptide corresponding to the aggrecan IGD domain as substrate (Supplementary Fig. S1).

\section{Interaction between fibulin-2 and ADAMTS-12 reduces breast cancer cell invasion and migration.}

Next, we wanted to assess the functional consequences of the interaction between ADAMTS-12 and fibulin-2. To this end, we transfected MCF-7 and MDAMB-231 cells with the full-length cDNAs for ADAMTS12 and FBLN2. Production of recombinant fibulin-2 (fb), ADAMTS-12 (ts) or both proteins simultaneously (fb/ts) was analyzed by Western blot (Fig. 2A and supplementary Fig. S2). Prior to functional analysis, we proved that presence of these extracellular proteins did not affect
A

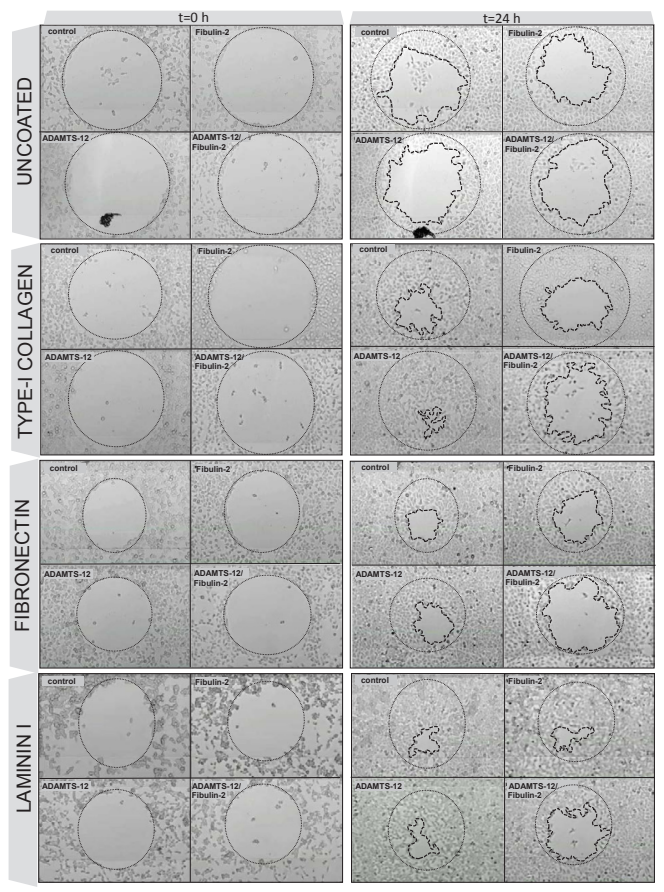

B

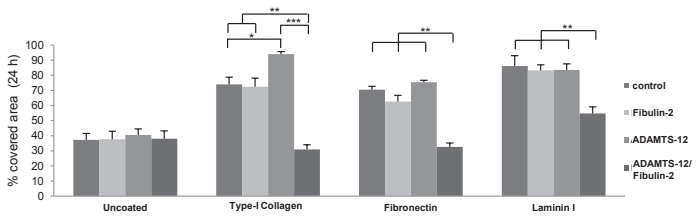

Figures 3: Interaction between fibulin-2 and ADAMTS-12 hinders migration of MCF-7 cells on ECM components. (A) MCF-7-(fb) (fibulin-2), MCF-7-(ts) (ADAMTS-12), and MCF-7-(fb/ts) (ADAMTS-12/fibulin-2) were allowed to migrate in uncoated wells or wells coated with type-I collagen, fibronectin or laminin I. MCF-7 cells transfected with an empty vector were used as control. Pictures of starting $(\mathrm{t}=0 \mathrm{~h})$ and final $(\mathrm{t}=24 \mathrm{~h})$ times are included. Starting point is indicated with a thin dotted line and final point with a thick dotted line. (B) Graphical representation of covered area after $24 \mathrm{~h}$ from three independent experiments. cell proliferation (Supplementary Fig. S3), and that the interaction between fibulin-2 and ADAMTS-12 also took place in MCF-7-(fb/ts) cells (Fig. 2B). Then, to analyze the role of this interaction in cell invasion, cells were allowed to invade using Matrigel-coated invasion chambers (Fig. $2 \mathrm{C})$. This assay showed that both MCF-7-(fb/ts) and MDA-MB-231-(fb/ts) cells exhibited a very low invasive potential in comparison with control cells $(63 \%$, and 76 $\%$ reduction respectively), or with cells producing only fibulin-2 (58\% and $47 \%$ reduction). Similarly to what has been previously reported [25], the highly invasive MDAMB-231 cell line producing fibulin-2, MDA-MB-231$(\mathrm{fb})$, remarkably reduced its invasive potential comparing to control cells (55\% reduction, Fig. $2 \mathrm{C})$. However, in the case of the poorly invasive MCF-7 no differences were observed between control and MCF-7-(fb) cells. By contrast, both MDA-MB-231-(ts) and MCF-7-(ts) showed a higher invasive ability than MDA-MB-231-(fb) and MCF-7-(fb) respectively (Fig. 2C).

Interaction between fibulin-2 and ADAMTS- 12 also affected cell migration. As can be seen in Fig. 3A, MCF7 -(fb/ts) cells did not show differences $(37 \%$ covered area) respecting MCF-7-(ts) (39\%), MCF-7-(fb) (37\%) or control cells ( $37 \%$ ) when migration was evaluated after $24 \mathrm{~h}$ using uncoated wells (supplementary videos 1 to 4 ).

A

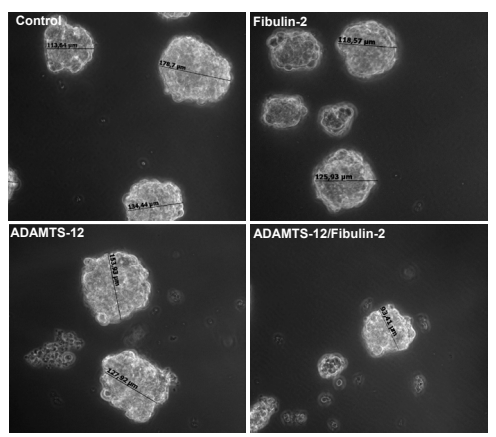

B

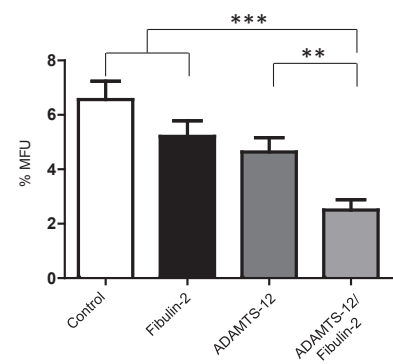

Figure 4: Simultaneous production of fibulin-2 and ADAMTS-12 reduces self-renewal of mammosphereforming units in MCF-7 cells. (A) Representative images of mammospheres derived from MCF-7-(fb) (fibulin-2), MCF-7-(ts) (ADAMTS-12), and MCF-7-(fb/ts) (ADAMTS-12/fibulin-2). Control, MCF-7 cells transfected with an empty vector. Sizes of some mammospheres are indicated. (B) Mammospheres were dissociated and passaged at a density of 20 cell/well in 96-well plates, and MFUs were counted and calculated as a percentage of mammospheres formed from the number of cell seeded. 
However, a significant reduction in migratory capacity of MCF-7-(fb/ts) cells was observed when these cells were grown in wells coated with fibronectin, laminin I, or type-I collagen (supplementary videos 5 to 8 ). Thus, $35 \%$ and $37 \%$ of area was covered by MCF-7-(fb/ts) cells in wells coated with type-I collagen and fibronectin, respectively. Nonetheless, MCF-7-(ts), MCF-7-(fb) and control cells covered $91 \%, 70 \%$ and $71 \%$ on type-I collagen, and 75 $\%, 62 \%$ and $70 \%$ on fibronectin respectively. In the case of laminin I, MCF-7-(fb/ts) cells covered $54 \%$ whereas MCF-7-(ts), MCF-7-(fb) and control cells covered around $85 \%$ (Fig. 3B). Similar experiments performed with MDA-MB-231 cells revealed that fibulin-2/ADAMTS-12 interaction also hindered the ability of these cells to migrate, but differences were not so pronounced between MDA-MB-231-(fb) and MDA-MB-231-(fb/ts) as in MCF7 cells (Supplementary Fig. S4). Altogether, these data support that ADAMTS-12 fibulin-2/ interaction hampers both invasion and migration of breast cancer cells in vitro, but also that ADAMTS-12 could exert pro-tumor effects in the absence of fibulin-2.

\section{Combined expression of FBLN2 and ADAMTS12 reduces mammosphere formation}

3D culture system mimics the natural tissue environment of the cell. In the case of breast cancer cells, this culture system allows mammosphere formation and reflects the ability of these cells for in vitro selfrenewal. Therefore we examined whether ADAMTS-12 and fibulin- 2 could also alter the mammosphere-forming ability of breast cancer cells. To this end, $4 \times 10^{4} \mathrm{MCF}-$ 7 cells were plated in 6-well plates and mammospheres were visualized after 7 days. MCF-7-(fb/ts) showed a smaller number and mammospheres size than MCF-7-(fb), MCF-7-(ts) or MCF-7 control cells (Fig. 4A and 4B). To quantify the mammosphere forming units (MFUs), cells were dissociated using trypsin and passaged at a density of 20 cells/well through a 96-well plate. 7 days later,
MFUs were calculated and MCF-7-(fb/ts) showed 3.2fold decrease of MFUs compared to MCF-7 control cells, and 2-fold decrease compared to MCF-7-(fb) and MCF7-(ts) cells (Fig. 4B). In the case of MDA-MB-231 cells, exogenous expression of ADAMTS-12 and fibulin-2 also caused a decrease in MFUs compared to MDA-MB-231(ts) cells (2-fold) and to MDA-MB-231 control cells (2.5fold). However, difference between MDA-MB-231-(fb/ts) and MDA-MB-231-(fb) was slight (Supplementary Fig. S5A and S5B). Overall, these results indicate that fibulin-2 and ADAMTS-12 affect ability of breast cancer cells to form mammospheres.

\section{Interaction between fibulin-2 and ADAMTS-12 inhibits tumor growth in vivo}

To evaluate the effect of ADAMTS12 and FBLN2 expression in vivo, MCF-7-(fb), MCF-7-(ts) or MCF7 -(fb/ts) cells were subcutaneously injected in the flank of nude mice. Control MCF-7 cells were injected in the opposite flank and the evolution of tumor growth was followed by 6 weeks. MCF-7-(fb/ts) cells displayed a significantly reduced tumor growth rate when comparing to MCF-7-(fb), MCF-7-(ts) or MCF-7 control cells (Fig. $5)$. Thus, at the time of sacrifice, tumor volume derived from MCF-7-(fb/ts) cells was, on average, $25 \%$ smaller than those derived from MCF-7-(fb) and MCF-7 control cells, and $90 \%$ smaller than those derived from MCF7 -(ts) cells. These results indicate that the interaction between fibulin-2 and ADAMTS-12 may also promote anti-tumor effects in vivo.

\section{Inverse correlation of ADAMTS12 and FBLN2 expression with breast cancer stage}

To determine clinical relevance of ADAMTS-12 and fibulin-2 in breast cancer, we performed an immunohistochemical analysis of a tissue array containing 16 samples of normal and breast cancer tissues. A strong
A

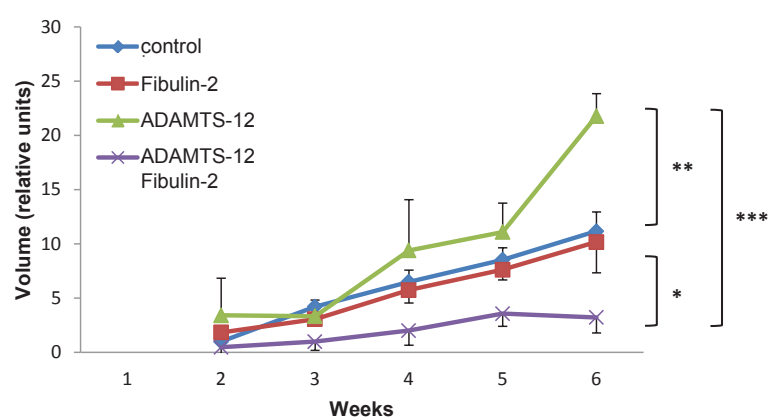

B

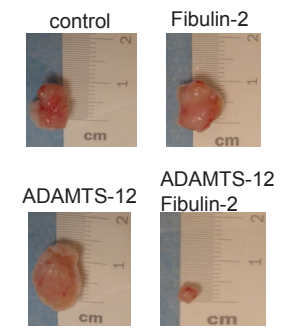

Figure 5: Presence of fibulin-2 and ADAMTS-12 inhibits growth of subcutaneous tumors. (A) Nude mice were injected with MCF-7-(fb) (fibulin-2), MCF-7-(ts) (ADAMTS-12), MCF-7-(fb/ts) (ADAMTS-12/fibulin-2) or control MCF-7 cells, and tumors growth were weekly measured. (B) Representative subcutaneous tumors at the time of sacrifice (6 weeks). 
staining for fibulin-2 was mainly detected in periductal connective tissue in normal breast (Fig. 6A). ADAMTS-12 was also detected in epithelia duct cells but showing a weaker staining. A moderate to strong expression of both fibulin-2 and ADAMTS-12 was detected in connective tissue surrounding tumor in grade 1 or 1 to 2 breast carcinoma $(n=5)$. However, weak or absent staining was observed in 10 out 11 (91\%) samples of grade 2 and 3 breast carcinoma for both fibulin-2 and ADAMTS-12. These data point to an inverse correlation between FBLN2 and ADAMTS12 co-expression and histophatological tumor grade.
We next assessed the association between ADAMTS12 and FBLN2 expression with clinical outcome using data available at www.kmplot.com. Kaplan-Meier curves revealed that a combined high expression of both ADAMTS12 and FBLN2 correlated with the best prognosis in breast cancer patients (Hts+Hfb, Fig. 6B) when compared to patients expressing low levels of both genes $(\mathrm{Lts}+\mathrm{Lfb})(p<0.001)$. This analysis also showed that simultaneous high ADAMTS12 and low FBLN2 expressions (Hts $+\mathrm{Lfb})$ associated to better outcome. However, high expression levels of FBLN2 combined with a low expression of ADAMTS12 (Lts+Hfb) showed
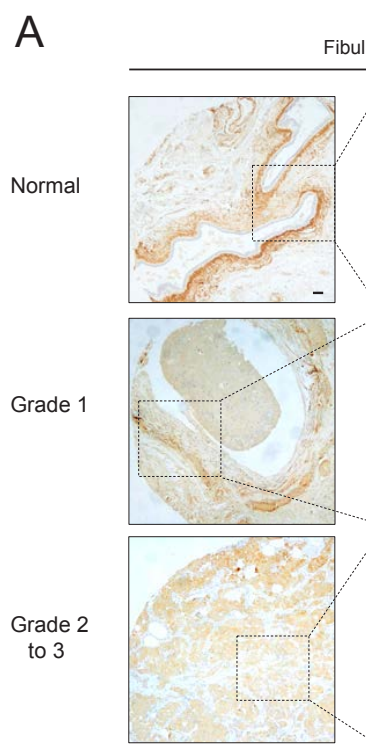

Grade 3

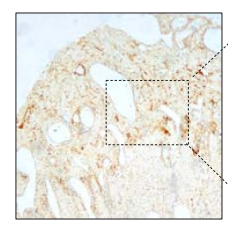

Fibulin-2
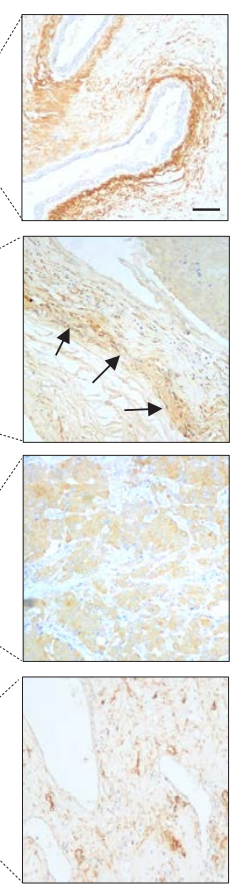
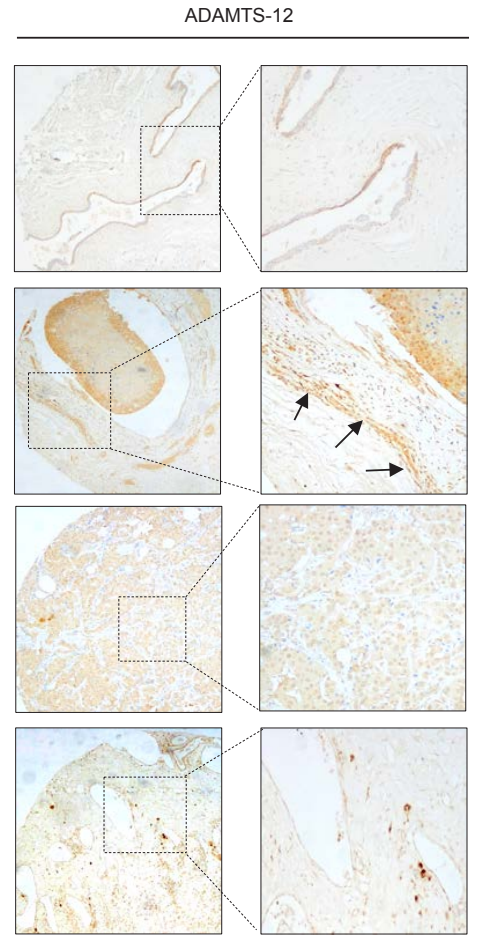

B

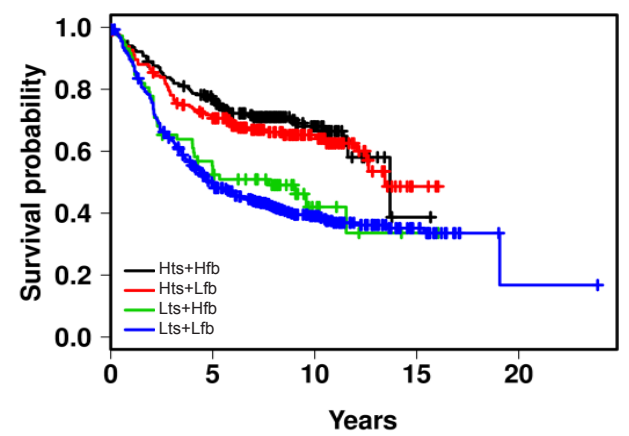

Figure 6: FBLN2 and ADAMTS12 expression analysis in breast cancer samples. (A) representative tissue images for detection of ADAMTS-12 and fibulin-2 in healthy breast tissue and breast carcinoma samples. Arrows indicate detection of ADAMTS-12 and fibulin-2 in grade 1 breast carcinoma. Scale bar, $200 \mu \mathrm{m}$. (B) Kaplan-Meier survival plots showing a better outcome of breast patients with high expression level of both ADAMTS12 and FBLN2 (Hts+Hfb). Plots for high ADAMTS12 and low FBLN2 (Hts+Lfb); low ADAMTS12 and high FBLN2 (Lts+Hfb); and low ADAMTS12 and low FBLN2 (Lts+Lfb) are also included. 
a slight but not significant increase of survival rate (Fig. $6 \mathrm{~B})$. These data suggests that factors other than combined expression of these two genes could modulate the pro or anti-tumor activities of both fibulin-2 and ADAMTS-12.

\section{DISCUSSION}

ECM regulates almost all cellular behavior and multiple regulatory mechanisms to ensure that production and remodeling of ECM components are normal during organ development $[1,35]$. Altered ECM can trigger cell signals or molecular interactions that promote cell survival or proliferation thereby contributing to cancer development [36]. Hence a major challenge in ECM biology is to understand the role of its components in normal and pathological processes. In this regard, a growing number of studies emphasize the multiple roles of fibulins in development and homeostasis of tissues [16]. Beyond this structural role, both tumor-suppressive functions and oncogenic activities have also been related to these glycoproteins [21]. Diverse mechanisms may underlie these apparently contradictory roles in cancer, including their interactions with other extracellular matrix components [13].

In the present work, using a yeast two-hybrid screening, we have found that fibulin-2 is a new partner of the ADAMTS-12 metalloprotease. Interaction between these two secreted proteins was confirmed by co-immunoprecipitation. Functional consequences of this interaction were evaluated by different cellbased assays using two human breast cancer cell lines, the poorly invasive $\mathrm{MCF}-7$ and the highly invasive MDA-MB-231. None of them express fibulin-2 [25] or ADAMTS-12 endogenously [30-31], hence we have selected clones producing ADAMTS-12, fibulin-2 or both proteins simultaneously. Exogenous expression of these proteins did not alter cell growth rates. However, results obtained from other cell-based assays point to two important findings. First, anti-tumor properties associated to fibulin-2 in breast cancer [25] are enhanced by its interaction with ADAMTS-12, especially in MCF-7 cells. Thus, production of both proteins remarkably reduced MCF-7 cells ability to invade in Matrigel-coated invasion chambers. This interaction also decreased the percentage of mammosphere-forming units, and strongly inhibited cell migration on fibronectin or laminin, two extracellular matrix components that associate to fibulin-2 [17]. Additionally, MCF-7-(fb/ts) cells displayed a reduced tumor growth when were subcutaneosuly injected in the flank of nude mice. Data obtained using MDA-MB-231 cells indicated that differences were less marked between MDA-MB-231-(fb/ts) and MDA-MB-231-(fb). In this regard, it has been previously found that pro-tumor properties of MDA-MB-231 cells are especially hampered by fibulin-2. In fact, exogenous expression of fibulin-2 in this cell line reduced speed of wound-healing closure and decreased cell invasion in vitro [25]. Moreover, fibronectin-induced migration of MDA-MB-231 cells is markedly inhibited by fibulin-1 [37], the closest molecular partner of fibulin-2. Consequently, both fibulin-1 and -2 may inhibit oncogenic properties of breast cancer cells. Mechanisms underlying these effects are not yet known, but association with other ECM components could also influence pro-tumor or anti-tumor activities of fibulins.

The second relevant finding is that ADAMTS-12 could promote pro-tumor properties when is produced in breast cancer cells in the absence of fibulin-2. Exogenous expression of the metalloprotease not only increased invasion and migration capacities of $\mathrm{MCF}-7$ cells in vitro but also produced significantly higher tumor volumes in nude mice. Contrary to this, we had previously found that exogenous expression of ADAMTS12 in A549 cells inhibited tumor growth in vivo [29]. An explanation for these apparent contradictory results is that A549 cells express fibulin-2 [14]. Thus, it can be speculated that exogenous ADAMTS-12 may interact with fibulin-2 produced by A549 cells and thereby inducing the antitumor effect observed. A previous report also supported the pro-tumor activity of this metalloprotease since its exogenous expression potentiated invasive capacity of the human placental choriocarcinoma cell line JEG3 [32]. Moreover, exogenous ADAMTS-12 reduced the ability of JEG-3 cells to bind to collagen-1 and our data indicate that presence of ADAMTS-12 increases migration on collagen-1 of MCF-7 cells. Interestingly, an enhanced stromal collagen-1 deposition correlates with breast cancer metastasis [38]. In this scenario and in the absence of fibulin-2, ADAMTS-12 could contribute to tumor progression by increasing the migratory capacity of breast tumor cells on collagen-1. By contrast, several studies have highlighted the anti-tumor role of this metalloprotease, including the phenotypic characterization of Adamts 12-deficient mouse [31]. Different mechanisms may cause the anti-tumor effects of ADAMTS-12. For instance, thrombospondin type-1 motifs at carboxyterminal region of the protease influence its antimetastatic potential. However this enzyme undergoes proteolytic processing events that could modify this effect [29]. Some of these properties resemble those previously described for ADAMTS-1. Thus, this metalloprotease was initially catalogued as a tumor-suppressor factor due to the angioinhibitory properties related to the thrombospondin type1 domains located at the carboxy-terminal region [39]. Furthermore, a decreased expression of ADAMTS-1 in breast cancer favors breast cancer migration and invasion [40]. Nonetheless, ADAMTS-1 can also generate anti-angiogenic peptides through the digestion of thrombospondin-1[41] or endorse the recruitment of fibroblasts involved in tumor growth, an effect also associated to an increased deposition of collagen-1 [42]. Moreover, ADAMTS-1 interacts with other member of the fibulin family, fibulin-1 [34], which in this case acts 
as a cofactor of the metalloprotease. Hence, fibulin-1 could potentiate degradation of ECM components by ADAMTS-1 and thereby facilitating tumor progression. We explored the possibility that fibulin- 2 could be a regulatory factor of the metalloprotease activity. Given that ADAMTS-12 displays low aggrecanase activity in vitro [29], we evaluated the digestion of a peptide corresponding to the human aggrecan interglobular domain. However, our results indicated that the presence of fibulin-2 did not potentiate the cleavage of this peptide by ADAMTS-12. Despite of this, it cannot be ruled out that this fibulin could act as an enhancer or inhibitor of ADAMTS-12 enzymatic activity in other circumstances considering the limited number of known in vivo substrates for this enzyme.

Immunohistochemical staining revealed that ADAMTS-12 was mainly detected in the stroma of grade 1 breast carcinomas, similar to what had been previously reported in colorectal cancer [30]. Staining pattern of fibulin-2 showed an equivalent situation. However, both proteins are hardly detected in grade 2 and 3 breast carcinomas. Thus, it can be speculated that a potential interaction between these two proteins may happen in less aggressive breast cancers and that this interaction could form part of a stromal response aimed to block cancer progression. Kaplan-Meier survival plots also suggested a potential tumor-suppressive role of the concomitant high expression of both ADAMTS12 and FBLN2. However, some results of this work also suggest a pro-tumor activity of ADAMTS-12 in the absence of fibulin-2. In consequence, pro or anti-tumor activities of ADAMTS-12 could also be influenced by factors other than interaction with fibulin-2.

Interaction between ADAMTSs and fibulins raises new questions about the role of these proteins in tumor progression. For instance, these interactions may underlie the molecular mechanisms by which some tumor-protective metalloproteases exert their anti-tumor activities. In this work we show that ADAMTS-12/ fibulin-2 interaction induces anti-tumor effects in breast cancer cells and our data also suggest that combined detection of both proteins could be a good prognosis marker in breast cancer patients. Further exhaustive functional and mechanistic studies will help finding out the precise role of ADAMTS-12 and fibulin-2 in relation to tumorigenesis, including the importance of the ADAMTS-12/fibulin-2 molar ratio as an indicator of potential invasiveness of breast cancer cells. Additionally, tumor susceptibility assays using mice lacking both Adamts 12 and Fbln2 genes would be necessary to understand in depth the significance of this interaction not only in breast cancer but also in tumors from different origins.

\section{MATERIALS AND METHODS}

\section{Yeast Two-hybrid Screening}

The Matchmaker Two-hybrid System and a human fetal kidney library (prey vectors) containing a sequence enconding amino acids $768-881$ of the GAL4 protein (AD) were purchased from Clontech. Screening process was performed following manufacturer's recommendations. Briefly, a cDNA fragment encoding amino acids 702813 of human ADAMTS-12 (GenBank ${ }^{\mathrm{TM}}$ accession number AAI31734) was PCR amplified using the primers 5'-AAACATATGCAGACTGTGAGAAAGAT GTT$3^{\prime}$ and 5'-TATGTCGACTGGATTGTGTACTCATA CTT-3' and inserted between the NdeI and SalI sites of the pGBKT7 vector to make a bait vector encoding a GAL4-BD-ADAMTS-12 $2^{702-813}$ chimeric protein. Both bait and prey vectors were introduced into Saccharomyces cerevisiae AH109 cells and selection of double transformants was performed on synthetic minimal medium lacking leucine, tryptophan and histidine. Colonies were restreaked, and prey plasmids rescued, transfected into Escherichia coli DH5 $\alpha$ and selected on LB medium containing $100 \mu \mathrm{g} / \mathrm{mL}$ ampicillin. Identification of the prey inserts was performed by direct sequencing.

\section{Human cell lines, plasmids and transfection}

MCF-7 and MDA-MB-231 breast cancer cells, obtained from ATCC, and 293-EBNA cells producing exogenous ADAMTS-12 containing a FLAG epitope [29] were routinely maintained in DMEM medium containing $10 \%$ heat-inactivated fetal bovine serum and $100 \mathrm{U} /$ $\mathrm{mL}$ penicillin and $50 \mu \mathrm{g} / \mathrm{mL}$ streptomycin. pCEP-TS12 vector [29] and the full-length cDNA clon HK9 [8] were employed to overproduce ADAMTS-12 and human fibulin-2 respectively. These vectors were transfected into MCF-7 and MDA-MB-231 cells using Lipofectamine reagent (Life Technologies) as recommended by the manufacturer. Recombinant ADAMTS-12 and fibulin-2 were obtained as previously described [8, 29]. Cells stably expressing ADAMTS12 (ts), FBLN2 (fb) or both simultaneously ( $\mathrm{fb} / \mathrm{ts})$, or transfected with an empty vector were selected in the presence of $1.5 \mu \mathrm{g} / \mathrm{mL}$ puromycin (Sigma-Aldrich).

\section{Western blotting and immunoprecipitation}

For western blot analysis, proteins were resolved by 8 or $12 \%$ polyacrylamide gel electrophoresis, transferred to a nitrocellulose membrane and subsequently probed with the indicated antibodies. Primary antibodies used for detection of ADAMTS-12 were H-142 (Santa 
Cruz Biotech) or anti-FLAG-M2 (Sigma-Aldrich), and for detection of fibulin-2 was H-250 (Santa Cruz Biotech). Anti-actin antibody was from Sigma-Aldrich. Immunoreactive proteins were visualized using HRPperoxidase labeled anti-rabbit or anti-mouse secondary antibody and the ECL detection system (Pierce). Relative expression levels were quantified and normalized to actin levels within the same blot using the Image $\mathrm{J}$ software. For immunoprecipitation, MCF-7-(fb/ts) were resuspended in lysis buffer $(20 \mathrm{mM}$ Tris- $\mathrm{HCl} \mathrm{pH} 8.0$, $150 \mathrm{mM} \mathrm{NaCl}, 5 \mathrm{mM}$ iodoacetamide, $0.5 \mathrm{mM} \mathrm{CaCl}_{2}$, $0.5 \mathrm{mM} \mathrm{MgCl}, 0.02 \% \mathrm{NaN} 3,1 \mathrm{mM}$ AEBSF and $1 \%$ Triton X-100), containing one complete protease inhibitor cocktail tablet (Roche Molecular Biochemicals) per 50 $\mathrm{mL}$ buffer. Protein concentration was quantified using the BCA Protein Assay Kit from Pierce Technology. To immunoprecipite ADAMTS-12, cell extracts were incubated with anti-FLAG M2 affinity gel (SigmaAldrich) following manufacturer's instructions. To immunoprecipitate fibulin-2, we used H-250 antibody and the Protein G Immunoprecipitation kit (Sigma-Aldrich), following manufacturer's instructions. MCF-7 cells extract producing fibulin-2 was used as a negative control. In the case of EBNA-293 cells producing ADAMTS-12, cell extracts were incubated with the indicated amounts of fibulin-2 prior to the immunoprecipitation step. EBNA cells transfected with an empty vector (EBNAc) were used as negative control.

\section{Enzyme activity}

ADAMTS-12 enzymatic activity was evaluated using a 10xHis tagged peptide corresponding to the interglobular (IGD) domain of aggrecan as substrate at $0.016 \mu \mathrm{g} / \mu \mathrm{l}$ (rhAggrecan G1-IGD-G2, R\&D Systems). As indicated, different amounts of fibulin-2 were added to the reaction. Reactions were performed in $20 \mu \mathrm{L}$ for 4 hours in the buffer previously described [29]. Degradation by ADAMTS-4 (R\&D Systems) was employed as positive control. Peptide degradation was examined by western blot using an anti-His antibody (Quiagen).

\section{Cell proliferation}

Cell proliferation was measured using the CellTiter 96 Non-radiactive Cell Proliferation Assay kit purchased from Promega. $10^{3}$ cells were seeded in 96-well plates and six replicates per condition and time point were assessed. Cell proliferation rates were determined on three (MCF7) or four (MDA-MB-231) consecutive days using an automated microtiter plate reader Power Wave WS (BioTek).

\section{Invasion assays}

In vitro invasion potential was evaluated using 24well Matrigel-coated invasion chambers with a $8 \mu \mathrm{m}$ pore size (BD Biosciences). For MCF-7 cells, $2.5 \times 10^{4}$ cells were allowed to migrate for $96 \mathrm{~h}$ using $10 \%$ foetal bovine serum as a chemoattractant. Cells that reached the lower surface were stained with crystal violet. At least three independent experiments were performed with triplicates for each condition. Cells were counted in eight randomly selected microscopic fields. In the case of MDA-MB-231 cells, invasion was evaluated after $24 \mathrm{~h}$.

\section{Migration assays}

Migratory capacity of cells on ECM components fibronectin, laminin I, and type-I collagen was examined using the Radius ${ }^{\mathrm{TM}}$ 24-Well Cell Migration Assay kit (Cell Biolabs) following manufacturer's instructions. Briefly, $1,25 \times 10^{4}$ cells were seeded per well and migration was monitored by time-lapse microscopy using a Zeiss Axio Observer Microscopy. Experiments were performed with triplicates and covered area was quantified at different times using Image J. Results were obtained after $24 \mathrm{~h}$ migration for MCF-7 cells and after $6 \mathrm{~h}$ for MDA-MB-231 cells.

\section{Mammospheres culture}

$4 \times 10^{4} \mathrm{MCF}-7$ or $5 \times 10^{3} \mathrm{MDA}-\mathrm{MB}-231$ cells were plated in 6-well ultralow attachment plates (Costar) and grown in MammoCult Basal Medium (Stem Cell Research) supplemented with $10 \%$ Mammocult Proliferation Supplement, $4 \mu \mathrm{g} / \mathrm{mL}$ heparine and $0.5 \mu \mathrm{g}$ / $\mathrm{mL}$ hydrocortisone. After 7 days, mammospheres were collected and enzymatically dissociated as previously described [43]. Individual dissociated cells were cultured in 96-well ultralow attachment plates at a density of 20 cells/well. Mammospheres formation was monitored microscopically daily to ensure they derived from single cells and not from aggregates. Number of mammospheres was determined after 7 days culture.

\section{Animals and subcutaneous tumors}

Four to five-week-old female athymic nude mice ( $n u / n u$ Swiss mice from Charles River Laboratories) were used for in vivo studies. To induce subcutaneous tumors, three groups of five mice were injected at one flank with 7 x $10^{6}$ cells of MCF-7-(fb), MCF-7-(ts) or MCF-7-(fb/ ts) cells in $200 \mu \mathrm{L}$ PBS. As a control, the opposite flank in each animal was injected with identical number of control MCF-7 cells transfected with an empty vector. Tumor growth was monitored weekly as previously described 
[29]. For estrogen supplementation, pellets of $1.5 \mathrm{mg}$ 17- $\beta$-estradiol, 90-day release (Innovative Research of America), were implanted between the scapulae at the time of injection. Mice were housed under specific pathogen-free conditions and following the guidelines of the Committee on Animal Experimentation of the Universidad de Oviedo, Asturias, Spain.

\section{Human breast cancer tissue array}

A breast cancer tissue array containing 16 tumors samples from different tumor stages (Acris Antibodies) was employed to evaluate ADAMTS12 and FBLN2 expression in human breast samples. Slides were processed for indirect peroxidase immunohistochemistry in the following way: sections were deparaffinized and rehydrated, and then rinsed in phosphate buffered saline (PBS) containing $1 \%$ tween-20. For detection of fibulin-2 (H-250 antibody) and ADAMTS-12 (H-142 antibody), sections were heated in high $\mathrm{pH}$ Envision FLEX target retrieval solution at $80{ }^{\circ} \mathrm{C}$ for $20 \mathrm{~min}$ and then incubated for $20 \mathrm{~min}$ at room temperature in the same solution. Endogenous peroxidase activity $\left(3 \% \mathrm{H}_{2} \mathrm{O}_{2}\right)$ and nonspecific binding ( $33 \%$ fetal calf serum) were blocked and the sections were incubated overnight at $4{ }^{\circ} \mathrm{C}$ with primary antibodies described above using a 1:100 dilution for both antibodies. As secondary antibodies we used labelled polymer-HRP ready to use from DAKO. 3-3' diaminobenzidine was employed as a chromogen. Selected slides were counterstained with haematoxylin. The intensity of immunostaining was evaluated by two independent observers directly upon microscope and was scored as absent, slight, moderate and strong. Study methodologies were conformed to the standards set by the Declaration of Helsinki and were approved by the Ethics Committee of the Universidad de Oviedo-Principado de Asturias (Spain).

\section{Survival analysis}

To assess the effect of ADAMTS-12 and fibulin-2 on breast cancer prognosis, survival probability was determined using the data available at www.kmplot.com [44]. 1592 patients were selected and we compared high (top $25 \%$ of patients) and low (bottom $25 \%$ of patients) expression of ADAMTS12, FBLN2 and both genes simultaneously. Results were represented as Kaplan-Meier long-rank test survival plots.

\section{Statistical analysis}

Statistical analysis were carried out using the GraphPad Prism 5.0 Software. Data were represented as means $+/$ - S.E. The occurrence of significant differences was determined with the Student-Welch $t$ test. $p$ values under 0.05 were considered statistically significant $(p<$ $0.05, * ; p<0.01, * * ; p<0.005, * * *)$.

\section{ACKNOWLEDGEMENTS}

We thank Dr. Carlos López-Otín for his comments and suggestions. This work was supported by a grant from the Instituto de Salud Carlos III (Spain) (FISS PI11/00371). The Instituto Universitario de Oncología is supported by Obra Social Cajastur.

\section{REFERENCES}

1. Lu P, Weaver VM and Werb Z. The extracellular matrix: a dynamic niche in cancer progression. J Cell Biol. 2012; 196(4):395-406.

2. Kalluri R. Basement membranes: structure, assembly and role in tumour angiogenesis. Nat Rev Cancer. 2003; 3(6):422-433.

3. Happonen KE, Heinegard D, Saxne T and Blom AM. Interactions of the complement system with molecules of extracellular matrix: relevance for joint diseases. Immunobiology. 2012; 217(11):1088-1096.

4. Vong S and Kalluri R. The role of stromal myofibroblast and extracellular matrix in tumor angiogenesis. Genes Cancer. 2011; 2(12):1139-1145.

5. Timpl R, Sasaki T, Kostka G and Chu ML. Fibulins: a versatile family of extracellular matrix proteins. Nat Rev Mol Cell Biol. 2003; 4(6):479-489.

6. Kobayashi N, Kostka G, Garbe JH, Keene DR, Bachinger HP, Hanisch FG, Markova D, Tsuda T, Timpl R, Chu ML and Sasaki T. A comparative analysis of the fibulin protein family. Biochemical characterization, binding interactions, and tissue localization. J Biol Chem. 2007; 282(16):1180511816.

7. Segade F. Molecular evolution of the fibulins: implications on the functionality of the elastic fibulins. Gene. 2010; 464(1-2):17-31.

8. Olin AI, Morgelin M, Sasaki T, Timpl R, Heinegard D and Aspberg A. The proteoglycans aggrecan and Versican form networks with fibulin-2 through their lectin domain binding. J Biol Chem. 2001; 276(2):1253-1261.

9. de Vega S, Iwamoto T, Nakamura T, Hozumi K, McKnight DA, Fisher LW, Fukumoto S and Yamada Y. TM14 is a new member of the fibulin family (fibulin-7) that interacts with extracellular matrix molecules and is active for cell binding. J Biol Chem. 2007; 282(42):30878-30888.

10. Pan TC, Kluge M, Zhang RZ, Mayer U, Timpl R and Chu ML. Sequence of extracellular mouse protein BM-90/fibulin and its calcium-dependent binding to other basementmembrane ligands. Eur J Biochem. 1993; 215(3):733-740.

11. Reinhardt DP, Sasaki T, Dzamba BJ, Keene DR, Chu ML, Gohring W, Timpl R and Sakai LY. Fibrillin-1 and fibulin-2 
interact and are colocalized in some tissues. J Biol Chem. 1996; 271(32):19489-19496.

12. Talts JF, Andac Z, Gohring W, Brancaccio A and Timpl $R$. Binding of the $G$ domains of laminin alphal and alpha2 chains and perlecan to heparin, sulfatides, alphadystroglycan and several extracellular matrix proteins. EMBO J. 1999; 18(4):863-870.

13. Obaya AJ, Rua S, Moncada-Pazos A and Cal S. The dual role of fibulins in tumorigenesis. Cancer Lett. 2012; 325(2):132-138.

14. Yue W, Sun Q, Landreneau R, Wu C, Siegfried JM, Yu J and Zhang L. Fibulin-5 suppresses lung cancer invasion by inhibiting matrix metalloproteinase-7 expression. Cancer Res. 2009; 69(15):6339-6346.

15. Hwang CF, Chien CY, Huang SC, Yin YF, Huang CC, Fang FM, Tsai HT, Su LJ and Chen CH. Fibulin-3 is associated with tumour progression and a poor prognosis in nasopharyngeal carcinomas and inhibits cell migration and invasion via suppressed AKT activity. J Pathol. 2010; 222(4):367-379.

16. de Vega S, Iwamoto T and Yamada Y. Fibulins: multiple roles in matrix structures and tissue functions. Cell Mol Life Sci. 2009; 66(11-12):1890-1902.

17. Utani A, Nomizu M and Yamada Y. Fibulin-2 binds to the short arms of laminin-5 and laminin-1 via conserved amino acid sequences. J Biol Chem. 1997; 272(5):2814-2820.

18. Sasaki T, Gohring W, Pan TC, Chu ML and Timpl R. Binding of mouse and human fibulin-2 to extracellular matrix ligands. J Mol Biol. 1995; 254(5):892-899.

19. Pfaff M, Sasaki T, Tangemann K, Chu ML and Timpl R. Integrin-binding and cell-adhesion studies of fibulins reveal a particular affinity for alpha IIb beta 3. Exp Cell Res. 1995; 219(1):87-92.

20. Argraves WS, Greene LM, Cooley MA and Gallagher WM. Fibulins: physiological and disease perspectives. EMBO Rep. 2003; 4(12):1127-1131.

21. Gallagher WM, Currid CA and Whelan LC. Fibulins and cancer: friend or foe? Trends Mol Med. 2005; 11(7):336340.

22. Senapati S, Gnanapragassam VS, Moniaux N, Momi N and Batra SK. Role of MUC4-NIDO domain in the MUC4mediated metastasis of pancreatic cancer cells. Oncogene. 2012; 31(28):3346-3356.

23. Law EW, Cheung AK, Kashuba VI, Pavlova TV, Zabarovsky ER, Lung HL, Cheng Y, Chua D, Lai-Wan Kwong D, Tsao SW, Sasaki T, Stanbridge EJ and Lung ML. Anti-angiogenic and tumor-suppressive roles of candidate tumor-suppressor gene, Fibulin-2, in nasopharyngeal carcinoma. Oncogene. 2012; 31(6):728-738.

24. Dunwell TL, Hesson LB, Pavlova T, Zabarovska V, Kashuba V, Catchpoole D, Chiaramonte R, Brini AT, Griffiths M, Maher ER, Zabarovsky E and Latif F. Epigenetic analysis of childhood acute lymphoblastic leukemia. Epigenetics. 2009; 4(3):185-193.
25. Yi CH, Smith DJ, West WW and Hollingsworth MA. Loss of fibulin-2 expression is associated with breast cancer progression. Am J Pathol. 2007; 170(5):1535-1545.

26. Cal S, Arguelles JM, Fernandez PL and Lopez-Otin C. Identification, characterization, and intracellular processing of ADAM-TS12, a novel human disintegrin with a complex structural organization involving multiple thrombospondin-1 repeats. J Biol Chem. 2001; 276(21):17932-17940.

27. Noel A, Gutierrez-Fernandez A, Sounni NE, Behrendt N, Maquoi E, Lund IK, Cal S, Hoyer-Hansen G and Lopez-Otin C. New and paradoxical roles of matrix metalloproteinases in the tumor microenvironment. Front Pharmacol. 2012; 3:140.

28. Luan Y, Kong L, Howell DR, Ilalov K, Fajardo M, Bai XH, Di Cesare PE, Goldring MB, Abramson SB and Liu CJ. Inhibition of ADAMTS-7 and ADAMTS-12 degradation of cartilage oligomeric matrix protein by alpha-2macroglobulin. Osteoarthritis Cartilage. 2008; 16(11):14131420.

29. Llamazares M, Obaya AJ, Moncada-Pazos A, Heljasvaara R, Espada J, Lopez-Otin C and Cal S. The ADAMTS12 metalloproteinase exhibits anti-tumorigenic properties through modulation of the Ras-dependent ERK signalling pathway. J Cell Sci. 2007; 120(Pt 20):3544-3552.

30. Moncada-Pazos A, Obaya AJ, Fraga MF, Viloria CG, Capella G, Gausachs M, Esteller M, Lopez-Otin C and Cal $\mathrm{S}$. The ADAMTS12 metalloprotease gene is epigenetically silenced in tumor cells and transcriptionally activated in the stroma during progression of colon cancer. J Cell Sci. 2009; 122(Pt 16):2906-2913.

31. El Hour M, Moncada-Pazos A, Blacher S, Masset A, Cal S, Berndt S, Detilleux J, Host L, Obaya AJ, Maillard C, Foidart JM, Ectors F, Noel A and Lopez-Otin C. Higher sensitivity of Adamts12-deficient mice to tumor growth and angiogenesis. Oncogene. 2010; 29(20):3025-3032.

32. Beristain $\mathrm{AG}, \mathrm{Zhu} \mathrm{H}$ and Leung PC. Regulated expression of ADAMTS-12 in human trophoblastic cells: a role for ADAMTS-12 in epithelial cell invasion? PLoS One. 2011; 6(4):e18473.

33. Porter S, Scott SD, Sassoon EM, Williams MR, Jones JL, Girling AC, Ball RY and Edwards DR. Dysregulated expression of adamalysin-thrombospondin genes in human breast carcinoma. Clin Cancer Res. 2004; 10(7):2429-2440.

34. Lee NV, Rodriguez-Manzaneque JC, Thai SN, Twal WO, Luque A, Lyons KM, Argraves WS and Iruela-Arispe ML. Fibulin-1 acts as a cofactor for the matrix metalloprotease ADAMTS-1. J Biol Chem. 2005; 280(41):34796-34804.

35. Wiseman BS, Sternlicht MD, Lund LR, Alexander CM, Mott J, Bissell MJ, Soloway P, Itohara S and Werb Z. Site-specific inductive and inhibitory activities of MMP2 and MMP-3 orchestrate mammary gland branching morphogenesis. J Cell Biol. 2003; 162(6):1123-1133.

36. Cox TR and Erler JT. Remodeling and homeostasis of the 
extracellular matrix: implications for fibrotic diseases and cancer. Dis Model Mech. 2011; 4(2):165-178.

37. Hayashido Y, Lucas A, Rougeot C, Godyna S, Argraves WS and Rochefort H. Estradiol and fibulin-1 inhibit motility of human ovarian- and breast-cancer cells induced by fibronectin. Int J Cancer. 1998; 75(4):654-658.

38. Zhang K, Corsa CA, Ponik SM, Prior JL, Piwnica-Worms D, Eliceiri KW, Keely PJ and Longmore GD. The collagen receptor discoidin domain receptor 2 stabilizes SNAIL1 to facilitate breast cancer metastasis. Nat Cell Biol. 2013; 15(6):677-687.

39. Vazquez F, Hastings G, Ortega MA, Lane TF, Oikemus S, Lombardo $\mathrm{M}$ and Iruela-Arispe ML. METH-1, a human ortholog of ADAMTS-1, and METH-2 are members of a new family of proteins with angio-inhibitory activity. J Biol Chem. 1999; 274(33):23349-23357.

40. Freitas VM, do Amaral JB, Silva TA, Santos ES, Mangone FR, Pinheiro Jde J, Jaeger RG, Nagai MA and MachadoSantelli GM. Decreased expression of ADAMTS-1 in human breast tumors stimulates migration and invasion. Mol Cancer. 2013; 12:2.

41. Lee NV, Sato M, Annis DS, Loo JA, Wu L, Mosher DF and Iruela-Arispe ML. ADAMTS1 mediates the release of antiangiogenic polypeptides from TSP1 and 2. EMBO J. 2006; 25(22):5270-5283.

42. Rocks N, Paulissen G, Quesada-Calvo F, Munaut C, Gonzalez ML, Gueders M, Hacha J, Gilles C, Foidart JM, Noel A and Cataldo DD. ADAMTS-1 metalloproteinase promotes tumor development through the induction of a stromal reaction in vivo. Cancer Res. 2008; 68(22):95419550.

43. Dontu G, Abdallah WM, Foley JM, Jackson KW, Clarke MF, Kawamura MJ and Wicha MS. In vitro propagation and transcriptional profiling of human mammary stem/ progenitor cells. Genes Dev. 2003; 17(10):1253-1270.

44. Gyorffy B, Benke Z, Lanczky A, Balazs B, Szallasi Z, Timar J and Schafer R. RecurrenceOnline: an online analysis tool to determine breast cancer recurrence and hormone receptor status using microarray data. Breast Cancer Res Treat. 2012; 132(3):1025-1034. 